\title{
ACTITUD HACIA LAS PERSONAS MAYORES Y VARIABLES ASOCIADAS EN UN GRUPO DE ESTUDIANTES UNIVERSITARIOS DEL AMBBITO DE LA EDUCACIÓN
}

\author{
González-Moreno, J. \\ jesus.gonzalezm@campusviu.es \\ Agusti, A.I. \\ Guillem, J. \\ Parra-Rizo, M.A. \\ Cantero-García, M.
}

Recepción Artículo: 06 diciembre 2021 Admisión Evaluación: 06 diciembre 2021 Informe Evaluador 1: 07 diciembre 2021 Informe Evaluador 2: 07 diciembre 2021 Aprobación Publicación: 07 diciembre 2021

\section{RESUMEN}

Las actitudes se definen como un constructo que implica un estado de disposición mental (aspectos cognitivos y afectivos) que influyen en nuestra percepción, pensamiento y motivación, y dirigen nuestras conductas. Las actitudes se convierten en elementos determinantes para predecir conductas positivas o negativas hacia los demás, especialmente hacia las personas mayores. El objetivo de este estudio es conocer las actitudes del alumnado de títulos universitarios de educación, ya que serán los próximos profesionales que eduquen. Conocer las actitudes y variables que puedan influir en estas puede ser un aspecto clave para desarrollar intervenciones posteriores, así como, para mejorar las actitudes hacia el mayor, y, por ende, su atención. Este estudio muestra que la actitud es moderadamente positiva, y que una actitud más positiva está relacionada con tener interés por temas de vejez, y con cuatro fortalezas humanas como son el amor, la bondad, la gratitud y la humildad.

Palabras clave: actitudes; adulto mayor; fortalezas; docentes

\section{ABSTRACT}

Attitude towards the elderly and associated variables in a group of university students in the field of education. Attitudes are defined as a construct that implies a state of mental disposition (cognitive and affective aspects) that influence our perception, thinking and motivation, and direct our behaviors. Attitudes become determinant elements in predicting positive or negative behaviors towards others, especially towards the elderly. The aim of this study is to know the attitudes of the students of university degrees in education, since they will be the next professionals to educate. Knowing the attitudes and variables that may influence them may be a key aspect for developing subsequent interventions, as well as for improving attitudes towards the elderly and, 


\section{ACTITUD HACIA LAS PERSONAS MAYORES Y VARIABLES ASOCIADAS EN UN GRUPO DE ESTUDIANTES UNIVERSITARIOS DEL ÁMBITO DE LA EDUCACIÓN}

therefore, their care. This study shows that the attitude is moderately positive, and that a more positive attitude is related to having interest in old age issues, and to four human strengths such as love, kindness, gratitude and humility.

Keywords: attitudes; older adult; strengths; teachers

\section{INTRODUCCIÓN}

El envejecimiento de la población, obliga a buscar la mejora de la atención hacia el colectivo de personas mayores, humanizando la atención y rechazando la discriminación de las personas mayores. En muchas ocasiones, una actitud negativa hacia la vejez y los prejuicios sobre esta población, merman la atención que reciben y los recursos que se les aportan (Barranquero y Ausín, 2019; Velasco et al., 2020). En los últimos años se ha visto un incremento de edadismo o discriminación por edad, cuestión que podría intervenirse si conociéramos que aspectos son determinantes a la hora de mostrar una serie de actitudes positivas.

\section{La importancia de las actitudes.}

A menudo se concibe a las personas mayores de manera estereotipada, asociándose esta etapa a estereotipos como: pasividad, aburrimiento, fragilidad, frustración, depresión, soledad e inutilidad (Fernández et al., 2017; OMS, 2019). Estas concepciones Ilevan al viejismo o discriminación sistemática contra las personas sólo por el hecho de ser mayores, desarrollándose actitudes negativas hacia el mayor.

Según los autores clásicos (Allaport,1935; Eagly y Chaiken, 1995) las actitudes se definen como un constructo que implica un estado de disposición mental (aspectos cognitivos y afectivos) que influyen en nuestra percepción, pensamiento y motivación, y dirigen nuestras conductas.

Para Fishbein y Azjen (1975) las actitudes pueden influenciar el comportamiento de las personas, si estas son positivas provocan pensamientos más positivos hacia los demás, por tanto, una mayor preocupación por sus necesidades, mejor atención y menor discriminación. Las actitudes se convierten en elementos determinantes para predecir conductas positivas o negativas hacia los demás, especialmente hacia las personas mayores (Fernández et al., 2017; Ortiz-Rubio et al., 2019; Sampen et al., 2012).

\section{La educación universitaria y las actitudes hacia el mayor.}

La actitud hacia las personas mayores se ve influenciada por la vida pasada del individuo, familia y creencias, valores culturales, nivel de educación y motivación (Dilek, 2011). Por tanto, la formación supone una oportunidad de poder intervenir y modificar estas actitudes. La mayoría de estudios previos se centran en las titulaciones de la salud puesto que la atención a personas mayores es directa (Fernández et al., 2017; Ortiz-Rubio, 2019). No obstante, un elevado número de docentes y alumnado, no sólo de ciencias de la salud o sociales, atiende o presta servicios a personas mayores ahora y cada vez más en el futuro, por ende, es de vital importancia conocer las actitudes hacia los mayores en el mundo universitario y poder elaborar un modelo de predicción, para posteriormente adoptar y desarrollar que mejores las actitudes hacia las personas mayores. Siempre teniendo en cuenta que los docentes son las personas encargadas de la educación formal de los niños y adolescentes, y la importancia de que tengan las herramientas necesarias para poder trasladar de forma transversal en sus asignaturas, la buena actitud hacia el adulto mayor.

\section{Las fortalezas personales.}

Las fortalezas y virtudes personales son rasgos individuales positivos, elementos que pueden ser claves a la hora de mostrar una serie de actitudes o comportamientos hacia los otros.

Desde el campo de la psicología positiva se define a las virtudes como aquellos rasgos centrales del "buen carácter", relacionados con una moralidad positiva y que se dan de forma relativamente universal (Noftle et al., 2011). De estas se desprenden las fortalezas definidas como las características psicológicas que se muestran en distintas situaciones, de forma estable y que tienen un valor moral (Peterson y Park, 2009). Se visualizan en los 
pensamientos, emociones, actitudes y conductas de la persona (Niemiec, 2010) y posibilitan a las personas dar lo mejor de sí mismas. (Wood et al., 2011). Estas características psicológicas pueden ser variables relacionadas a la hora de mostrar una serie de actitudes, además estas pueden ser aprendidas y/o entrenadas mediante la educación/aprendizaje.

Por todo lo anterior, este proyecto tiene como objetivos describir cómo son las actitudes hacia el adulto mayor en estudiantes universitarios del ámbito de educación (futuro profesorado) y explorar la relación entre variables sociodemográficas y personales, con las actitudes hacia el mayor en estudiantes universitarios ámbito de la educación (futuro profesorado).

\section{MÉTODO}

Se trata de un estudio cuantitativo, ex post facto y estrategia prospectiva. Con un solo tiempo de medida. La muestra se extraerá mediante muestreo por conveniencia entre la población universitaria de titulaciones afines. La recogida de datos se llevará a cabo mediante una encuesta online.

Se llevaron a cabo análisis descriptivos/exploratorios de las principales variables, también se llevaron a cabo análisis correlaciones. Se utilizaron para los cálculos estadísticos necesarios el programa SPSS versión 25.

El presente proyecto se rige por los principios de la DECLARACIÓN DE HELSINKI, recogiendo el consentimiento informado de los participantes.

\section{Muestra}

Se recabaron un total de 69 encuestas de estudiantes universitarios del ámbito de la educación. Un 81,8\% fueron mujeres frente a un $18,2 \%$ de hombres. El $73,9 \%$ conviven con mayores y 76,8\% mostraban mucho 0 algo de interés por temas relacionados con la vejez y la gerontología.

\section{Instrumentos}

Cuestionario de sociodemográficos: que permita conocer las principales características de la población en cuanto a sexo y experiencia en la atención/cuidado de personas mayores.

Escala de Actitudes hacia el Adulto Mayor de Kogan (KAOP, Sampén et al., el año 2012). Para medir las actitudes hacia los adultos mayores se utilizará la versión validada en población hispanoamericana en lengua española del instrumento. Que consta de 34 enunciados divididos en dos categorías unos aspectos positivos y en correspondencia con estos en igual cantidad aspectos negativos, evaluados con una escala de Likert de 5 puntos, este instrumento obtuvo una fiabilidad de 0.82 determinada por el Alfa de Conbrach durante el proceso de validación. Además, es un instrumento frecuentemente utilizado en la investigación y de uso actual como por ejemplo en el trabajo de Henríquez et al. (2020).

Global Assessment of Character Strengths- 24 (GACS-24; VIA Institute on Character,2017) instrumento para evaluar las 24 fortalezas personales mediante autocuestionario compuesto por 24 ítems con siete niveles de respuesta que van de 1 (muy en desacuerdo) a 7 (muy de acuerdo). En este estudio se utilizó la versión en español. La fiabilidad media de sus elementos fue de $=.78$ (McGrath, 2017). Este instrumento está diseñado para la investigación y ha sido recientemente usado en otras investigaciones como la de Umucu et al. (2020).

\section{RESULTADOS}

Genéricos:

El 87\% de los sujetos manifestaron tener a alguna persona mayor de 65 años en su familia, no obstante, sólo el 73,9\% había convivido con alguna persona mayor.

En cuanto al interés relacionados con temas de la vejez, el 76\% muestra algo o mucho interés (tabla 1). 
Tabla 1. Porcentajes interés en temas relacionados con la vejez

Mucho 42

$\begin{array}{ll}\text { Algo } & 34,8 \\ \text { Poco } & 16,9\end{array}$

Muy poco $\quad 7,2$

No obstante, el interés en formarse en el ámbito es menor que el interés mostrado en temas relacionados en la vejez (tabla 2)

Tabla 2. Porcentajes interés en formarse en temas relacionados con la vejez

Mucho 34,8

$\begin{array}{ll}\text { Algo } & 31,9 \\ \text { Poco } & 14,5\end{array}$

Muy poco 4,3

De media la muestra tiene una experiencia profesional en el campo de 5 años $(D T=7,6)$, pero tan sólo 3,6 años $(D T=3,6)$ de media de experiencia en estudios relacionados con el ámbito.

\section{RESULTADOS EN ACTITUD Y FORTALEZAS}

En la escala de actitudes hacia el mayor se obtuvo una puntuación media de 146,2 (DT =17,48), lo que muestra una actitud que tiene tendencia a la positividad, pero pudiendo ser mejorable (ya que la puntuación máxima posible es de 204). Se obtuvo una puntuación mínima de 109 y una máxima de 196.

En lo que respecta a las fortalezas, en esta muestra destaca el amor por el aprendizaje ( $M=6,1 ; D T=1$ ) y la que menos se da es la de espiritualidad ( $M=4,1 ; D T=1,9)$.

Por último, en lo que respecta a las relaciones entre las variables, se ha encontrado relación significativa en las siguientes variables (Tabla 3 ).

Tabla 3. Correlación actitud positiva con fortalezas y otras variables.

\begin{tabular}{llllll}
\hline & Amor & Bondad & Gratitud & Humildad & $\begin{array}{l}\text { Interés por temas } \\
\text { vejez }\end{array}$ \\
\hline $\begin{array}{l}\text { Actitud } \\
\text { más positiva }\end{array}$ & ,352** & ,328** & ,292* & ,246* & ,238* \\
\hline
\end{tabular}

La correlación significativa en el nivel 0,01 (bilateral).**. Correlación significativa en nivel 0,05 (bilateral).*

Siendo, por tanto, el amor, la bondad, la gratitud y la humildad fortalezas relacionadas con una actitud más positiva hacia las personas mayores. También el interés por temas de la vejez estaría asociado con una puntuación mayor en actitud positiva hacia las personas mayores. 


\section{DISCUSIÓN Y CONCLUSIONES}

En línea de otros trabajos con estudiantes universitarios en otros ámbitos (Fernández et al., 2017; Ortiz-Rubio et al., 2019), se ha observado que existe una tendencia hacia una actitud positiva en esta población, pero esta puede ser mejorada. Por tanto, encontramos capacidad para intervenir y aumentar esas actitudes positivas hacia las personas mayores en estudiantes universitarios del área de educación. Otros estudios que han encontrado actitudes negativas por parte de estudiantes universitarios en países como México o Chile (Fernández et al., 2016, Medina et al., 2021) apuntan que la presencia de estereotipos negativos sobre la vejez afecta a las actitudes, y plantean que la formación puede ser una vía para su mejora. En el presente trabajo se muestra el interés por formarse en temas relacionados con la gerontología o el envejecimiento está relacionado con actitudes más positivas hacia el mayor, por tanto, la formación se erige como una oportunidad para reducir los prejuicios sobre la vejez y aumentar la actitud positiva hacia las personas mayores.

Por otro lado, sabemos que existen una serie de características psicológicas que se muestran en distintas situaciones, de forma estable y que tienen un valor moral (Io que conocemos como fortalezas humanas). Y que estas aparecen a través de las actitudes (Niemiec, 2010). Por ello, uno de los objetivos del presente trabajo era conocer cuáles son las fortalezas más destacadas en esta muestra y su relación con la actitud hacia los mayores. En este caso se ha encontrado que las fortalezas de amor, bondad, gratitud y humildad tienen una relación positiva con una mayor puntuación en la escala de actitud hacia las personas mayores, lo que significa que existe una actitud más positiva. Según Ovejero et al. (2016) las fortalezas pueden ser potenciadas, por tanto, potenciar las fortalezas del amor, la bondad, la gratitud y la humildad en estudiantes universitarios del ámbito de la educación, podrá ayudar a mejorar las actitudes hacia las personas mayores.

El presente estudio, pese a que tiene poca muestra y no ha estudiado el nivel formativo de los participantes como principales limitaciones, supone un relevante avance. La mayoría de trabajos se centra en estudiar cómo son las actitudes, pero no se paran a analizar qué aspectos podrían ayudar a mejorarlas. La literatura que estudia la relación de las fortalezas humanas y las actitudes hacia las personas mayores es prácticamente inexistente, por tanto, este trabajo debe continuar, estudiarse en otros contextos y muestras para poder desarrollar intervenciones de mejora de las actitudes en un futuro.

\section{REFERENCIAS BIBLIOGRÁFICAS}

Allport, G. W. (1935). Attitudes. En C. Murchison (Ed.), Handbook of Social Psychology (pp. 798-884). Worcester, MA: Clark University Press.

Barranquero, R., y Ausín, B. (2019). Impacto de los estereotipos negativos sobre la vejez en la salud mental y física de las personas mayores. Psicogeriatría, 9(1), 41-47. Disponible en:

https://www.researchgate.net/publication/334454554_Impacto_de_los_estereotipos_negativos_sobre_la_ vejez_en_la_salud_mental_y_fisica_de_las_personas_mayores

Eagly, A. H. \& Chaiken, S. (1995). Attitude strength, attitude structure, and resistance to change. En R. E. Petty \& J. A. Krosnick (Eds.), Attitude strength: antecedents and consequences (pp. 413-432). Mahwah, NJ: Lawrence Erlbaum.

Fernández, E., Padilla, P., Monardes, H., y Díaz, C. (2017). Actitud hacia el adulto mayor en estudiantes del pregrado de la Facultad de odontología Universidad San Sebastián, Santiago. Revista Estomatológica Herediana, 27(1), 21-29. http://dx.doi.org/10.20453/reh.v27i1.3099

Fishbein, M. y Ajzen, I. (1975). Belief, Attitude, Intention And Behavior: An introduction to theory and research. Addison-Wesley

Henríquez, F., Retamal, N., Silva, F., \& Morales, C.. (2020). Actitudes hacia el envejecimiento por parte de los estudiantes de Fonoaudiología de una Universidad Chilena. CoDAS, 32(1), e20190010. https://doi.org/10.1590/2317-1782/20192019010 
McGrath, R. E. (2017). Technical report: The VIAassessment suite for adults: Development and ini-tial evaluation. VIA Institute on Character. https://www.viacharacter.org/pdf/Technical\%20Report\%20Revised\%20Edition\%202019_1.pdf

Medina, I. A., Medina, J. A., Torres, R., Sosa, R., Chale, G. W. y Chaparro, L. (2021). Actitudes hacia la vejez y actitudes hacia la sexualidad del adulto mayor en estudiantes y profesionales de enfermería. Gerokomos, 32(1), 17-21. Recuperado de https://dx.doi.org/10.4321/s1134-928x2021000100005

Niemiec, R. M. (2010). Then principles of character strengths. Positive Psychology News Daily. Disponible en: http://positivepsychologynews.com/news/ryan-niemiec/2010052611161.

Noftle, E. E., Schnitker, S. A., y Robins, R. W. (2011). Character and personality: Connections between Positive Psychology and Personality Psychology. En K.M. Sheldon, T. B. Kashdan y M. F. Steger (Eds.), Designing Positive Psychology. Taking Stock and Moving Forward (pp 207-227). Nueva York: Oxford University Press.

Organización Mundial de la Salud- OMS (2019). Informe Mundial sobre el Envejecimiento y la Salud. Ginebra: Organización Mundial de la Salud. Disponible en:

https://apps.who.int/iris/bitstream/handle/10665/186466/9789240694873_spa.pdf

Ortiz-Rubio, A., Cabrera-Martos, I., Torres-Sánchez, I., Casilda-López, J., Ariza-Mateos, M. J., \& Valenza, M. C. (2019). Actitudes de estudiantes españoles de terapia ocupacional hacia las personas mayores. Revista de la Facultad de Medicina, 67(1), 69-73.

Ovejero Bruna, M. M., Cardenal Hernáez, V., \& Ortiz-Tallo, M. (2016). Fortalezas humanas y bienestar biopsicosocial: Revisión Sistemática. Escritos de Psicología (Internet), 9(3), 4-14. https://dx.doi.org/10.5231/psy.writ.2016.2311

Peterson, C., y Park, N. (2009). El estudio científico de las fortalezas humanas. En C. Vázquez y G. Hervás (coords.), La Ciencia del Bienestar. Fundamentos de una Psicología Positiva (pp. 181-207). Madrid. Alianza Editorial.

Sampén, J. D., Varela, L. F., Díaz, C., Tello,T., y Ortiz, P. J. (2012). Validación de la Escala de Actitudes hacia el Adulto Mayor de Kogan y evaluación de las Actitudes hacia el adulto mayor por parte del personal de salud del primer nivel asistencial. Acta Médica Peruana, 29(3), 148-154. Disponible en: http://www.scielo.org.pe/scielo.php?script=sci_arttext\&pid=S1728-59172012000300004\&lng=es\&tlng=es

Umucu, E., \& Lee, B. (2020). Examining the impact of COVID-19 on stress and coping strategies in individuals with disabilities and chronic conditions. Rehabilitation psychology, 65(3), 193-198. https://doi.org/10.1037/rep0000328

Velasco, V., Suárez, G., Limones, M., Reyes, H., y Delgado, V. (2020). Creencias, estereotipos y prejuicios del adulto mayor hacia el envejecimiento. European Journal Of Health Research, 6(1), 85-96. https://doi.org/10.30552/ejhr.v6i1.204

VIA Institute on Character (2017). Global Assessment of Character Strengths- 24. VIA Institute on Character. https://www.viacharacter.org/researchers/assessments/global-assessment-of-character-strengths-24

Wood, A. M, Linley, A., Maltby, J., Kashdan, T. B., y Hurling, R. (2011). Using personal and psychological strengths leads to increases in well-being over time: A longitudinal study and the development of the strengths use questionnaire. Personality and Individual Differences, 50, 15-19. https://doi.org/10.1016/j.paid.2010.08.004 